\title{
Study of a wake up radio architecture for home multimedia networks
}

\author{
Aissa Khoumeri, Florin Hutu, Guillaume Villemaud, Jean-Marie Gorce
}

\begin{abstract}
A theoretical study on the impact of using a wake-up radio architecture in terms of energy consumption is proposed. The main objective is to reduce the overall energy consumption of a home multimedia networks. The energy consumed by the proposed wake-up radio architecture is compared to a classical WiFi architecture, for an ad-hoc scenario. The sleep time has an important role to compare the dissipated energy. This study demonstrate that the longer the sleep time the better the energy saved is obtained by the wake-up architecture.
\end{abstract}

\section{Introduction}

Nowadays wireless local area networks (WLANs) are widely deployed to provide access to the internet. Even at home, the number of devices to be connected to the internet increases and consequently the energy consumption of the home multimedia network grows. The necessity to reduce the energy consumption of the connected devices is ongoing challenge for researchers in order to develop a green home wireless network. Worldwide consortium's gathering academic and industrial partners formed and gave themselves, as challenges to increase the energy efficiency by a factor of 1000 in the next few years.

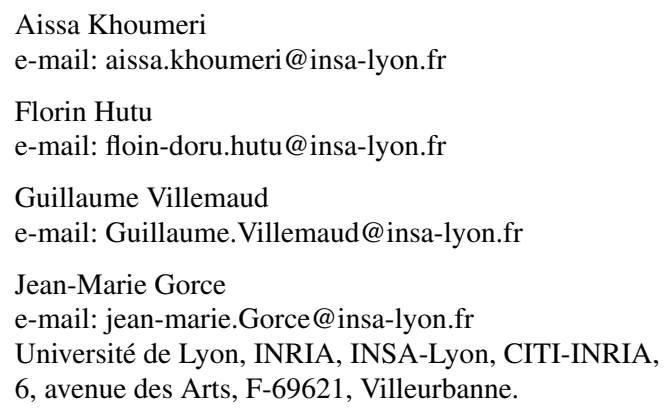


An approach to reduce the energy consumption of the home multimedia network is to shut down the RF part of the main data interfaces when there is no communication demand, for example the "power save mode" used in IEEE 802.11 [1].

The energy consumed by the radio interface in the idle mode remains relatively high because of the low energy efficiency of the RF front end. For example, in the case of Linksys WRG54G Wi-Fi access point (AP), the maximum reduction in the energy consumption that can be achieved is $28.57 \%$ [2]. This value represents the energy consumption of Wi-Fi interface compared to the entire energy consumption of the access point.

The wake-up concept has been introduced in the context of wireless sensor networks (WSN) as a way to optimize the battery lifetime [3] [4] [5] [6]. In WSN, the duty-cycle MAC protocols to reduce the overall power consumption exists. Wireless sensors in a duty cycle mechanism, should periodically sleep and wake their radio modules (like S-MAC, T-MAC, and B-MAC [7] [8] [9] [10]). High duty cycle reduce the latency but increases the energy consumption while low duty cycle reduces the energy consumption but increases the latency. An appropriate trade-off between energy consumption and latency has to be achieved.

Another mechanism of the wake-up radio is to use a secondary low power transceiver to monitor the channel instead of the main data transceiver. In this paper, the terms "Wake-up architecture" designs both the secondary low power and the main data transceivers.

Usually, the On Off Keying (OOK) modulation and the non-coherent envelope detection technique are used to design the low power secondary transceivers. This is because of their simplicity to implement, and their low energy consumption.

The use of a wake-up radio in the home wireless network, like personnel digital assistant (PDAs), has been firstly presented in [11]. In [2]. A low power sleep mode and an out of band wake-up mechanism has been adopted, a low power radio module to carry out of band control information is used to switch the WiFi AP into the sleep mode when no users exist.

The use of secondary low power wireless module, which has the same frequency and shares the same antenna with the co-located WiFi radio interface, was proposed in [12]. Based on modulation of the frame length of IEEE $802.11 \mathrm{~g}$, the wake-up signal recognition need an analogue to digital converter and a signal detection module that introduce more power consumption to the wake-up receiver.

In our previous work [13] [14] we present a wake-up radio architecture based on a frequency pattern identification of the addressed equipment. The secondary low power wireless module does not need baseband treatment of the identifier and the decision to wake up is taken directly in the analog part. This implies a reduction of the energy consumption in sleep mode and reduction of latency. In this paper an evaluation of benefits of using such architecture in terms of energy consumption is given.

The rest of this paper is organized as follows. Section 2 presents the proposed wake-up architecture and the identification technique. Section 3 presents the energy consumption estimation in an ad-hoc scenario. Finally, Section 4 presents some conclusions and future works. 


\section{Wake-up radio architecture description}

The proposed wake-up architecture in [13] [14] has an ultra-low power secondary wake-up radio circuit that monitors the channel for the wake-up signal identifier. In order to minimize the energy consumption, the wake-up receiver is designed to be simple as possible, as seen in Figure 1.

The Wake-up circuit has two paths in which, the input signal passes, (i) the direct path which contains the multi-band filter that has the same bandwidth as the spectrum formed by the identifier signal Figure 2, (ii) the complementary path contains the complementary multi-band filter, which has its pass bandwidth situated in the band stop of the direct filter. This configuration allows the wake-up radio circuit to eliminate the interference signal generated by other equipment present in the same area.

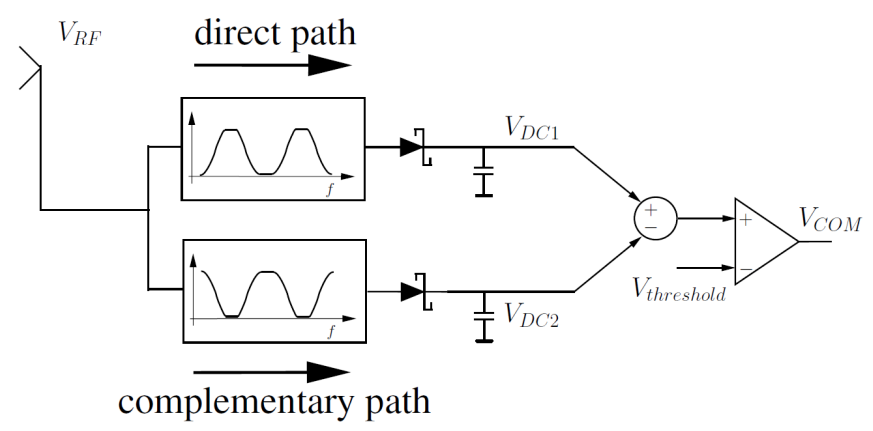

Fig. 1: Wake-up radio circuit.

The identifier is formed with an arrangement of a WLAN power spectrum, obtained by a selection of specific OFDM sub-carriers of the same group $G_{i}$ in the channel. Figure 2 shows an example of an identifier power spectrum. The 64 subcarriers in the total band of $20 \mathrm{MHz}$ are divided into 4 groups. Each group contains 14 neighbor sub-carrier of $312.5 \mathrm{KHz}$ as shown below:

$$
\left\{\begin{array}{r}
G_{1}=\{-28 \ldots-15\} \\
G_{2}=\{-14 \ldots-1\} \\
G_{3}=\{1 \ldots 14\} \\
G_{4}=\{15 \ldots 28\}
\end{array}\right.
$$

Because of the complementary configuration of the architecture, the same DC voltage is obtained in the two paths when another signal is received for example the WLAN RF signal, in this case the difference of the two DC voltages is null. 


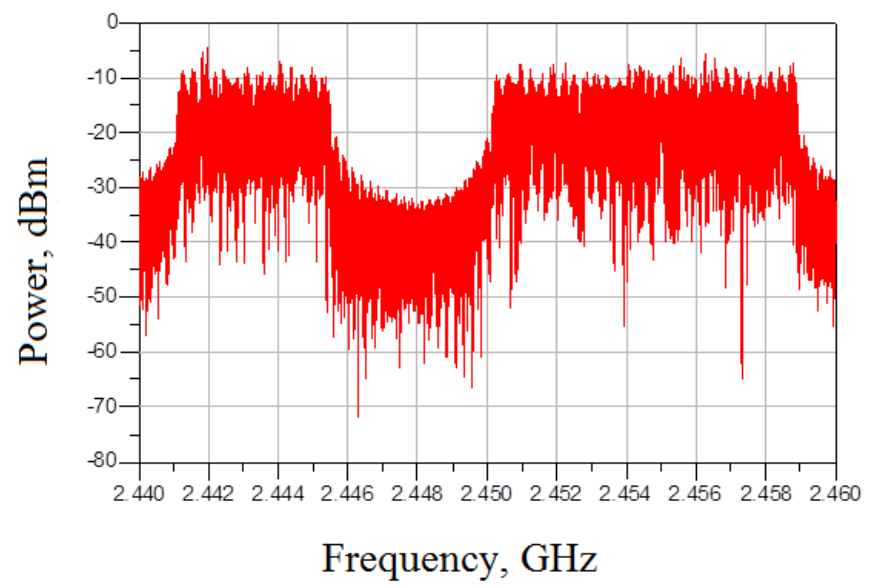

Fig. 2: Power spectrum of an identifier $G_{1}=\mathrm{ON} G_{3}=\mathrm{ON} G_{4}=\mathrm{ON}$.

When the identifier is received, the output DC voltage VDC1 obtained from the rectifier has the maximum value and the DC voltage VDC2 is null. In this case, the difference between the two DC voltages is higher than $V_{\text {threshold }}$, and the activation signal $V_{C O M}$ is generated as shown in Figure 3.

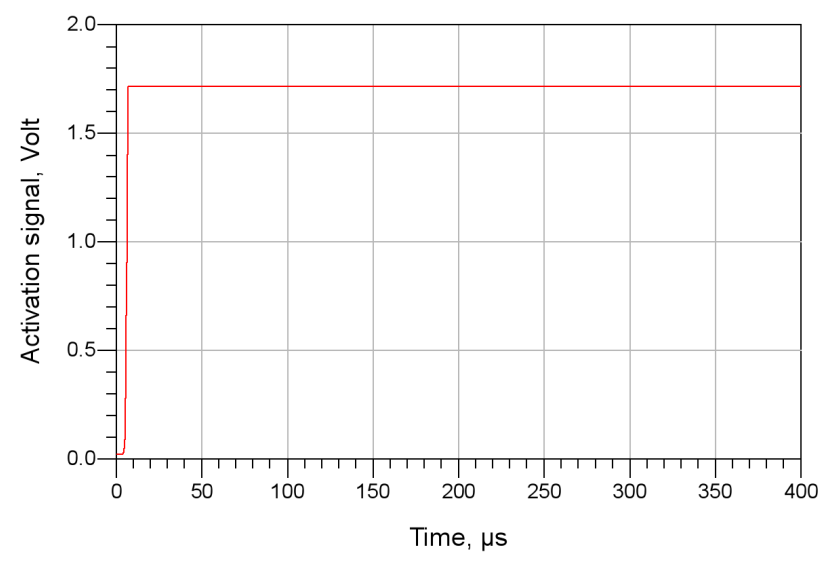

Fig. 3: Wake-up signal activation $V_{C O M}$. 


\section{Energy consumption estimation}

In order to calculate the energy consumption in a Wake-up radio architecture. We need to consider the consumption of the wake up circuit and the main data radio interface in different states of communication. In this study ad-hoc scenario as shown in Figure 4 is considered. Equipment 2 has its main interface "Main data 2" off, and its secondary wake-up radio receiver monitor the channel. When the wake-up signal identifier is sent by the equipment 1 , then the equipment 2 passes from offline state to the receiving state. And the data transfer between the two main interfaces may begin.

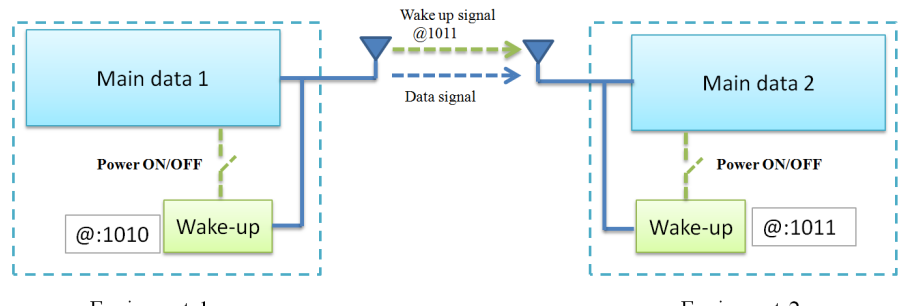

Equipment 1

Equipment 2

Fig. 4: proposed Wake-up radio architecture in an ad-hoc scenario.

The main data interface used in the study is the 2.4-2.5 GHz transceiver MAX2830 [15]. Figure 5 gathers the different timing state and power consumption of this frontend. This timing state start when the wake-up radio circuit receives the $400 \mu s$ identifier signal.

In the case of a classical WiFi the MAC controls the state of the physical layer (PHY). It achieves this by following the distributed coordinated function (DCF) access method [1]. All stations (STA) that intend to transmit frames must monitor the channel to determine if another STA is transmitting.

If the channel is idle for an interval of time that exceeds the distributed interframe space (DIFS), then the packet is transmitted. Otherwise, the STA monitor the channel until it is sensed idle for a DIFS interval. After that it generate a random backoff interval chosen in the range of $[0, \mathrm{CW}]$, where $\mathrm{CW}$ is the minimum contention window $\left(C W_{\text {min }}\right)$. Next the STA will reduce this number by one each time an idle slot is elapses, or it is frozen when the medium is sensed busy.

The energy consumption $E_{W L A N}^{\text {active }}$ to transmit and receive one frame by the classical WiFi architecture is given by (2). Also the energy consumed in the case of a wake up radio architecture $E_{\text {Wake-up }}^{\text {active }}$ is given by (3).

Table 1 defines the relevant parameters used in the energy model. These parameters are taken from the MAC layer of a standard 802.11g, based on OFDM PHY. And the power consumption in different states (Transmitting, receiving, idle and sleep ) of the MAX2830 transceiver. 


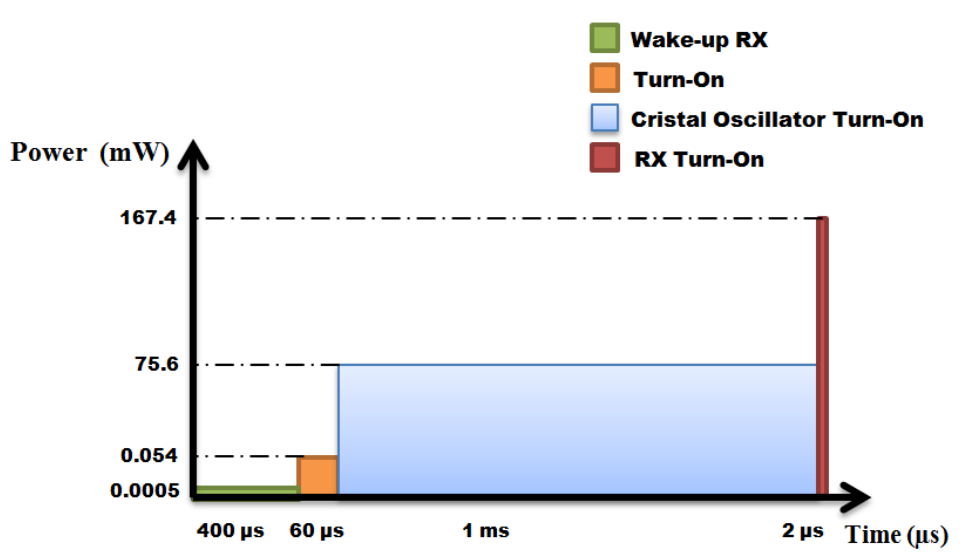

Fig. 5: Time and energy spend to wake up with MAX2830.

Equation (4) defines the energy consumed by the classical WiFi for sending and receiving one data frame for a classical WiFi architecture :

$$
E_{W L A N}^{a c t i v e}=\left(P_{T X}+P_{R X}\right) \times\left(T_{\text {data }}+T_{A C K}\right)+P_{\text {idle }}\left(2 \times T_{S I F S}+T_{D I F S}+T_{\text {Backoff }}\right)
$$

Equation (5) defines the energy consumed in the wake-up architecture for sending and receiving one data frame :

$$
\begin{array}{r}
E_{\text {Wake-up }}^{\text {active }}=\left(E_{\text {WLAN }}^{\text {active }}-P_{\text {idle }}\left(T_{\text {DIFS }}+T_{\text {Backoff } f}\right)\right)+\left(P_{T X}+P_{\text {wake-up }}\right) T_{\text {wake-up }} \\
+P_{\text {Turn-On }} \times T_{\text {Turn- } 0 n}+P_{\text {oscil }} \times T_{\text {oscil }}+P_{R X} \times T_{R X-O n}
\end{array}
$$

The energy consumed in sleep mode by the classical WiFi architecture is given by :

$$
E_{W L A N}^{\text {sleep }}=2 \times P_{\text {sleep }} \times T_{\text {sleep }}
$$

The energy consumed in sleep mode by the wake-up architecture is given :

$$
E_{\text {Wake-up }}^{\text {sleep }}=2 \times P_{\text {wake-up }} \times T_{\text {sleep }}
$$

The total energy consumed by the classical WiFi is given by :

$$
E_{W L A N}^{\text {total }}=E_{W L A N}^{\text {active }}+E_{W L A N}^{\text {sleep }}
$$

The total energy consumed by the wake-up architecture is given by :

$$
E_{\text {Wake-up }}^{\text {total }}=E_{\text {Wake-up }}^{\text {active }}+E_{\text {Wake-up }}^{\text {sleep }}
$$




\begin{tabular}{|l|l|r|}
\hline Variable & description & value \\
\hline$P_{T X}$ & Power consumed in transmitting mode & $800 \mathrm{~mW}$ \\
\hline$P_{R X}$ & Power consumed in reception mode & $167.4 \mathrm{~mW}$ \\
\hline$P_{\text {idle }}$ & Power consumption in standby mode & $75.6 \mathrm{~mW}$ \\
\hline$P_{\text {sleep }}$ & Power consumed in sleep mode & $75.6 \mathrm{~mW}$ \\
\hline$P_{\text {wake }- \text { up }}$ & Power consumed by the wake up secondary circuit & $500 \mathrm{nW}$ \\
\hline$P_{\text {Turn-On }}$ & Power consumed by the radio interface in shutdown mode & $54 \mu \mathrm{W}$ \\
\hline$P_{\text {oscil }}$ & Power consumed by the frequency synthesizer block & $75.6 \mathrm{~mW}$ \\
\hline$T_{\text {data }}$ & Time spend for sending 1500 byte of data at 9 Mbps & $2.42 \mathrm{~ms}$ \\
\hline$T_{\text {SIFS }}$ & Time of short interframe space & $10 \mu \mathrm{s}$ \\
\hline$T_{\text {DIFS }}$ & Time of distributed interframe space & $28 \mu \mathrm{s}$ \\
\hline$T_{\text {Backoff }}$ & Time of backoff & $67.5 \mu \mathrm{s}$ \\
\hline$T_{\text {wake-up }}$ & Time of wake up identifier & $400 \mu \mathrm{s}$ \\
\hline$T_{\text {Turn-0n }}$ & Time to pass from off mode to shutdown mode & $60 \mu \mathrm{s}$ \\
\hline$T_{R X-O n}$ & Time to pass from standby mode to receive mode & $2 \mu \mathrm{s}$ \\
\hline$T_{\text {sleep }}$ & Time spend in sleep state & Variable \\
\hline
\end{tabular}

Table 1: Parameters for modeling the energy consumption using a MAX2830 802.11g transceiver.

Figure 6 shows the energy consumption of the classical WiFi equation (6) and the wake-up architecture equation (7) when sending and receiving one frame of 1500 bytes with a rate of 9 Mbps.

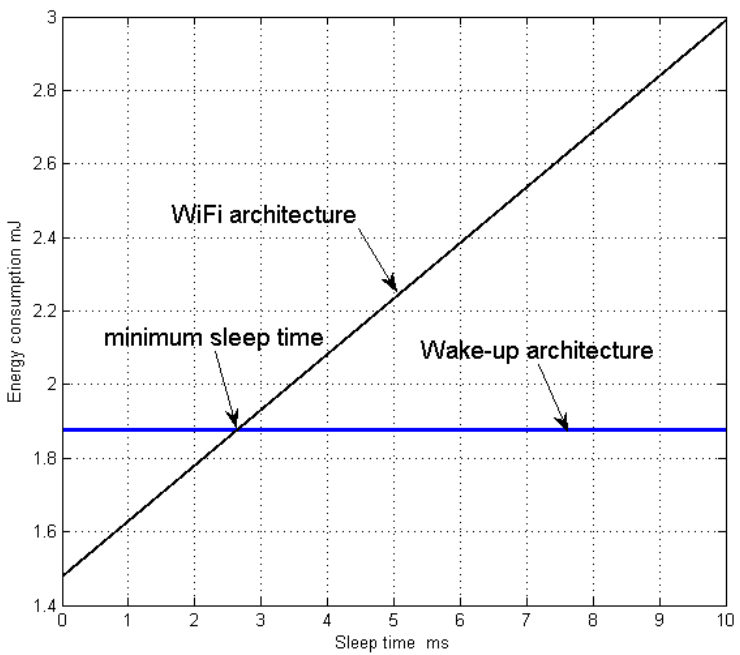

Fig. 6: Energy consumption vs sleep time. 
In equation (8) $T_{\text {sleep }}^{\min }$ represent the sleep time where the wake-up architecture has the same energy consumption as a classical WiFi architecture. From equations (6) and (7) we get this minimum time $T_{\text {sleep }}^{\text {min }}$ :

$$
T_{\text {sleep }}^{\text {min }}=\frac{1}{2} \times \frac{E_{\text {Wake-up }}^{\text {total }}-E_{\text {WLAN }}^{\text {total }}}{P_{\text {sleep }}-P_{\text {wake-up }}}
$$

If the sleep time is less than $T_{\text {sleep }}^{\text {min }}=2.6 \mathrm{~ms}$ the energy consumed by the wake-up architecture is higher than the energy consumed by the classical WiFi architecture. This due to the energy consumed by the main data when switching from offline to the receiving mode.

When the sleep time is higher than $T_{\text {sleep }}^{\min }$, the energy consumption of wake-up architecture is less than a classical WiFi architecture, because of it low energy consumption in sleep mode $500 \mathrm{nW}$.

\section{Conclusion}

In this paper, the energy consumption of the proposed wake-up radio architecture has been evaluated, and compared to a classical WiFi. The study demonstrate that with a respect minimum sleep time, the wake-up architecture save more energy than the classical WiFi. Future Works includes analyzing a network scenario, with a traffic rate (on the order of hours) to find the maximum sleep time in different traffic loads. However in such scenario latency and false wake up should be studied in order to quantify the robustness of the proposed architecture. Moreover a prototype will be manufactured in order to have measurement.

\section{References}

1. "IEEE standard for information technology and information exchange between systems - local and metropolitan area networks - specific requirements - part 11: Wireless lan medium access control (mac) and physical layer (phy) specifications," IEEE Std 802.11, pp. 1-1238, 2007.

2. I. Haratcherev, M. Fiorito, and C. Balageas, "Low-power sleep mode and out-of-band wake-up for indoor access points," in GLOBECOM Workshops, 2009 IEEE, pp. 1-6, IEEE, 2009.

3. L. Gu and J. A. Stankovic, "Radio-triggered wake-up for wireless sensor networks," Real-Time Systems, vol. 29, no. 2-3, pp. 157-182, 2005.

4. J. Ansari, D. Pankin, and P. Mähönen, "Radio-triggered wake-ups with addressing capabilities for extremely low power sensor network applications," International Journal of Wireless Information Networks, vol. 16, no. 3, pp. 118-130, 2009.

5. M. S. Durante and S. Mahlknecht, "An ultra low power wakeup receiver for wireless sensor nodes," in Sensor Technologies and Applications, 2009. SENSORCOMM'09. Third International Conference on, pp. 167-170, IEEE, 2009.

6. A. Khoumeri, F. Hutu, G. Villemaud, and J.-M. Gorce, "Wake-up radio architectures used in wireless sensor networks," in COST IC1004, (Lyon, France), May 2012. 
7. W. Ye, J. Heidemann, and D. Estrin, "An energy-efficient mac protocol for wireless sensor networks," in INFOCOM 2002. Twenty-First Annual Joint Conference of the IEEE Computer and Communications Societies. Proceedings. IEEE, vol. 3, pp. 1567-1576, IEEE, 2002.

8. T. Van Dam and K. Langendoen, "An adaptive energy-efficient mac protocol for wireless sensor networks," in Proceedings of the 1st international conference on Embedded networked sensor systems, pp. 171-180, ACM, 2003.

9. J. Polastre, J. Hill, and D. Culler, "Versatile low power media access for wireless sensor networks," in Proceedings of the 2nd international conference on Embedded networked sensor systems, pp. 95-107, ACM, 2004.

10. N. Ullah, P. Khan, and K. S. Kwak, "A very low power mac (vlpm) protocol for wireless body area networks," Sensors, vol. 11, no. 4, pp. 3717-3737, 2011

11. E. Shih, P. Bahl, and M. J. Sinclair, "Wake on wireless: an event driven energy saving strategy for battery operated devices," in Proceedings of the 8th annual international conference on Mobile computing and networking, pp. 160-171, ACM, 2002.

12. S. Tang, H. Yomo, Y. Kondo, and S. Obana, "Wake-up receiver for radio-on-demand wireless lans," EURASIP Journal on Wireless Communications and Networking, vol. 2012, no. 1, pp. 1-13, 2012.

13. A. Khoumeri, F. D. Hutu, G. Villemaud, and J.-M. Gorce, "Proposition d'une architecture de réveil radio utilisée dans le contexte des réseaux multimédia domestiques," in Journées Nationales Microondes, (Paris, France), May 2013.

14. F. Hutu, A. Khoumeri, G. Villemaud, and J.-M. Gorce, "Wake-up radio architecture for home wireless networks," in Radio and Wireless Symposium (RWS), 2014 IEEE, (Newport Beach, USA), January 2014. in press.

15. MAXIM, MAX2830 Industry's $2.4 \mathrm{GHz}$ to $2.5 \mathrm{GHz} 802.11 \mathrm{~g} / \mathrm{b}$ RF Transceiver, $P A$, and Rx/Tx/Antenna Diversity Switch, april 2008. 